\title{
Sex-dependent association between erythrocyte n-3 PUFA and type 2 diabetes in older overweight people
}

\author{
Kylie A. Abbott ${ }^{1}$, Martin Veysey ${ }^{2}$, Mark Lucock $^{3}$, Suzanne Niblett ${ }^{2}$, Katrina King ${ }^{2}$, Tracy Burrows ${ }^{1}$ and \\ Manohar L. Garg ${ }^{4 *}$ \\ ${ }^{1}$ School of Health Sciences, University of Newcastle, NSW 2308, Australia \\ ${ }^{2}$ School of Medicine \& Public Health, University of Newcastle, NSW 2308, Australia \\ ${ }^{3}$ School of Environmental E Life Sciences, University of Newcastle, NSW 2308, Australia \\ ${ }^{4}$ Nutraceuticals Research Group, School of Biomedical Sciences E Pharmacy, University of Newcastle, NSW 2308, Australia \\ (Submitted 24 August 2015 - Final revision received 13 December 2015 - Accepted 28 December 2015 - First published online 18 February 2016)
}

\section{Abstract}

The association between $n$-3 PUFA intake and type 2 diabetes (T2D) is unclear, and studies relating objective biomarkers of $n$ - 3 PUFA consumption to diabetic status remain limited. The aim of this study was to determine whether erythrocyte $n$ - 3 PUFA levels ( $n$ - 3 index; $n$ - 3 I) are associated with T2D in a cohort of older adults ( $n$ 608). To achieve this, the $n$-3I (erythrocyte \%EPA $+\% \mathrm{DHA})$ was determined by GC and associated with fasting blood glucose; HbA1c; and plasma insulin. Insulin resistance (IR) was assessed using the homeostatic model assessment of insulin resistance (HOMA--IR). OR for T2D were calculated for each quartile of $n$-3I. In all, eighty-two type 2 diabetic ( $46.3 \%$ female; 76.7 (sD 5.9) years) and 466 non-diabetic (57.9\% female; 77.8 (sD 7.1) years) individuals were included in the analysis. In overweight/ obese (BMI $\geq 27 \mathrm{~kg} / \mathrm{m}^{2}$ ), the prevalence of T2D decreased across ascending $n$-3I quartiles: $1 \cdot 0$ (reference), $0 \cdot 82$ (95\% CI 0.31, $\left.2 \cdot 18\right), 0 \cdot 56(95 \%$ CI $0.21,1.52)$ and $0.22(95 \%$ CI $0 \cdot 06,0.82)\left(P_{\text {trend }}=0.015\right)$. A similar but non-significant trend was seen in overweight men. After adjusting for BMI, no associations were found between $n$-3I and fasting blood glucose, HbA1c, insulin or HOMA-IR. In conclusion, higher erythrocyte $n$ - 3 PUFA status may be protective against the development of T2D in overweight women. Further research is warranted to determine whether dietary interventions that improve $n$-3 PUFA status can improve measures of IR, and to further elucidate sex-dependent differences.

Key words: $\boldsymbol{n}$-3 Index: Type 2 diabetes: Obesity: PUFA: Insulin resistance

Obesity is strongly associated with the development of insulin resistance (IR) and subsequent progression to type 2 diabetes (T2D), a growing public health problem, with worldwide prevalence expected to more than double between 2000 and $2030^{(1)}$. Dietary consumption of $n$-3 PUFA, particularly marine-based EPA (20:5n-3) and DHA (22:6n-3), has been shown to be effective in controlling dyslipidaemias and inflammation in diabetics ${ }^{(2)}$; however, the role of $n-3$ PUFA in the prevention of T2D is unclear.

A Cochrane review reported that $n-3$ PUFA supplements did not affect glycaemic control in T2D; however, a non-significant reduction in fasting insulin levels was noted ${ }^{(3)}$. Prospective cohort studies investigating $n$-3 PUFA intake and diabetes show mixed findings ${ }^{(4-8)}$. An ecological study investigating data across forty-one countries found that, in countries with a high prevalence of obesity, those with a higher intake of fish and seafood products had a lower prevalence of T2D than those with lower fish intake, showing a potentially weight-dependent effect $^{(9)}$.

To date, most studies of the relationship between $n$-3 PUFA levels and risk of T2D have only investigated dietary intake of $n-3$ PUFA, with few incorporating biochemical markers of $n-3$ PUFA intake. The $n-3$ index (n-3I), defined as the sum of EPA and DHA present in the erythrocyte membranes expressed as a percentage of the total fatty acids, has been documented to be a reliable marker of long-term dietary $n$ - 3 PUFA intake and is a good indicator of tissue levels ${ }^{(10)}$. The $n$ - 3 I has been shown to be negatively correlated with IR in obese children ${ }^{(11)}$; however, the relationship between $n-3$ PUFA status and T2D in older persons is unknown. The aim of this study was to determine whether $n-3$ PUFA status is associated with T2D in older Australians, and to test the hypothesis that the $n-3 \mathrm{I}$ is negatively correlated with IR in obese older adults.

\section{Methods}

\section{Subjects}

The present study was a sub-study of the Retirement Health and Lifestyle Study (RHLS), a cross-sectional study of older Australians living in the Central Coast of NSW, Australia.

Abbreviations: HOMA-IR, homeostatic model assessment of insulin resistance; IR, insulin resistance; $n$-3I, $n$-3 index; RHLS, Retirement Health and Lifestyle Study; T2D, type 2 diabetes.

* Corresponding author: Professor M. L. Garg, fax +61 24921 2028, email manohar.garg@newcastle.edu.au 
Participants were eligible for the RHLS study if they were $\geq 65$ years; if their primary residence was located within the Wyong or Gosford Local Government Areas; and if they had been living in their current address for $\geq 12$ months. Exclusion criteria for the RHLS included not living independently or residing in a communal setting other than a retirement village; if another member of their household was taking part in the study; having language and/or other communicative difficulties that limited participation; being cognitively impaired; and/or unable to provide informed consent. Participants were included in the present study if they had anthropometric measures and blood samples collected; if their diabetic status could be determined; and if they did not report having type 1 diabetes or insulin dependence. This research was conducted according to the Declaration of Helsinki. Participants provided written informed consent, and ethics approval for the study was obtained from the University of Newcastle Human Research Ethics Committee (reference no. H-2008-0431) and the Northern Sydney Central Coast Health Human Research Ethics Committee (reference no. 1001-031M). An overview of participant recruitment is presented in Fig. 1.

\section{Demographics and medical history}

Demographic data were collected in an intervieweradministered questionnaire (IAQ). Self-administered questionnaires were used to collect information on medical history and medication/supplement use.

\section{Dietary intake and physical activity}

Nutrient intake was determined using a self-administered semiquantitative FFQ, which was adapted from a validated $\mathrm{FFQ}^{(12)}$. Dietary intake was analysed using FoodWorks Professional Edition 2009 version 6.0.2562 (Xyris software), and mean daily energy $(\mathrm{kJ} / \mathrm{kcal})$ and macronutrient intakes were calculated using the following databases: Abbott products, AusFoods (brand) 2006, AusNut (all foods) 2007, Australia (fatty acids) and The New Zealand Vitamin and Mineral Supplement 1999.

Physical activity was assessed as part of the IAQ using questions that measured the frequency, duration and intensity of physical activity undertaken in the previous week. Questionnaire items captured information about time spent doing household chores and gardening; gentle exercise such as walking; moderate and vigorous physical activity; and strength training. Responses were used to categorise participants according to their level of physical activity: sedentary, low, moderate or vigorous. The questions used were adapted from validated surveys measuring physical activity ${ }^{(13-15)}$.

\section{Anthropometrics}

Anthropometric measures were taken by trained research officers. Height was measured with shoes off, to the nearest $\mathrm{mm}$ using a portable stadiometer (design no. 1013522 Surgical and Medical Products). Weight in light clothing and without shoes was measured to the nearest $100 \mathrm{~g}$ on digital scales (Tanita HD-316 (Tanita Health Equipment H.K. Ltd) and Wedderburn UWPM150 (W.W. Wedderburn Pty Ltd)). Waist circumference was measured with a non-elastic flexible measuring tape at the

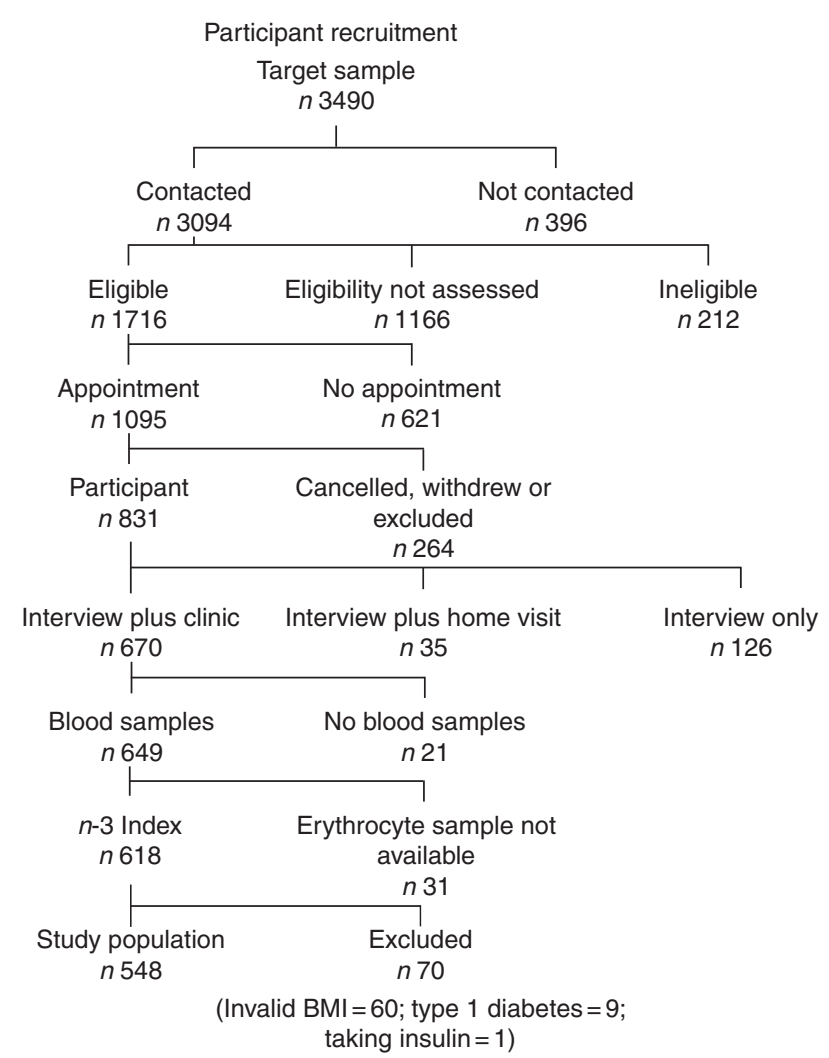

Fig. 1. Overview of participant recruitment.

mid-point between the iliac crest and the costal margin in the mid-auxiliary line, with the participant in the standing position. All measures were taken twice, with a third measure taken if there were discrepancies outside of specified tolerance limits. Height and weight were used to calculate BMI $\left(\mathrm{kg} / \mathrm{m}^{2}\right)$. BMI was used to categorise participants into a healthy/underweight group $\left(\mathrm{BMI}<27 \mathrm{~kg} / \mathrm{m}^{2}\right)$ and an overweight/obese group $\left(\mathrm{BMI} \geq 27 \mathrm{~kg} / \mathrm{m}^{2}\right)$. This BMI cut-off was selected as evidence suggests that in adults aged $>70$ years the risk of all-cause mortality is only increased for individuals with BMI over $27 \mathrm{~kg} / \mathrm{m}^{2(16)}$.

\section{Blood samples and biochemical analysis}

Blood samples were collected by a trained phlebotomist following a minimum of a 10-h overnight fast. Blood samples were analysed by Hunter Area Pathology Service for fasting insulin, fasting blood glucose and HbA1c levels using standardised laboratory protocols. Fasting insulin (mU/l) and blood glucose levels (BGL) ( $\mathrm{mmol} / \mathrm{l})$ were used to calculate homeostatic model assessment of insulin resistance (HOMA-IR) scores (fasting insulin $\times$ fasting glucose/22.5).

Fatty acid composition was analysed by GC based on methods established by Lepage and Roy ${ }^{(17)}$. Erythrocyte pellets were washed three times by adding $5 \mathrm{ml}$ of distilled water, centrifuging at $3000 \mathrm{~g}$ at $4^{\circ} \mathrm{C}$ for $10 \mathrm{~min}$ and discarding the supernatant to obtain erythrocyte membranes. $2 \mathrm{ml}$ of methanol-toluene mix (4:1 by volume) with C19:0 as an internal standard was added to the membrane pellet. While vortexing, $200 \mu$ of acetyl chloride was added drop-wise and the sample was heated at 
$100^{\circ} \mathrm{C}$ for $60 \mathrm{~min}$. Samples were cooled to room temperature by running under distilled water, and the reaction was stopped by adding $5 \mathrm{ml}$ of $6 \% \mathrm{~K}_{2} \mathrm{CO}_{3}$; the sample was vortexed to mix and then centrifuged at $3000 \boldsymbol{g}$ for $10 \mathrm{~min}$ to separate the layers. The upper layer containing toluene and fatty acid methyl esters was transferred to a 2-ml glass vial, and the vial crimp was sealed with a teflon-lined cap for GC analysis.

Methylated fatty acid samples were analysed by GC using a fixed carbon-silica column $30 \mathrm{~m} \times 0.25 \mathrm{~mm}$ (DB-225) (J and W Scientific). The GC was equipped with a flame ionisation detector, autosampler and autodetector. Injector and detector ports were set at $250^{\circ} \mathrm{C}$. Oven temperature was programmed for $170^{\circ} \mathrm{C}$ for $2 \mathrm{~min}$, and then increased $10^{\circ} \mathrm{C} / \mathrm{min}$ up to $190^{\circ} \mathrm{C}$ where it remained stationary for $1 \mathrm{~min}$. Temperature was then increased $3^{\circ} \mathrm{C} / \mathrm{min}$ up to $220^{\circ} \mathrm{C}$, which was maintained for a total run time of $30 \mathrm{~min}$ per sample. A split ratio of 10:1 and an injection volume of $3 \mu \mathrm{l}$ was used. A known fatty acid mixture was used to identify peaks according to retention time. Fatty acid concentration was determined using a Hewlett Packard 6890 Series GC with Chemstations version A. 04.02.

The $n$-3I was calculated by summing the erythrocyte membrane EPA and DHA, and the result was expressed as a percentage of the total fatty acids present ${ }^{(18)}$. Diabetic status was defined by participants having a fasting blood glucose $>7.0 \mathrm{mmol} / \mathrm{l}$ or taking diabetic medication ${ }^{(19)}$. IR was categorised according to HOMA-IR score $>3 \cdot 8^{(20)}$.

\section{Statistical analysis}

Data were assessed for normality, with the majority of data normally distributed, and thus parametric analyses were used. Descriptive statistics were calculated for all participants (total group), by sex, diabetic status (diabetic $v$. non-diabetic) and weight status (overweight/obese $v$. healthy/underweight).
Differences between groups were assessed using independent sample $t$ tests. Participants were categorised into quartiles according to their $n$-3I. Differences in the proportion of people with T2D and IR across the quartiles of $n$-3I were reported as OR (95\% CI), using the lowest quartile as a reference. Bivariate and multivariate relationships between fasting plasma insulin, fasting plasma glucose, HbA1c, HOMA-IR scores and $n$-3I were analysed using Pearson's correlations and hierarchical regression analyses. Statistical analyses were conducted using SPSS software version 17.0 (SPSS Inc.). Statistical significance was set as $P<0.05$. All values were reported as the mean values and standard deviation.

\section{Results}

A total of 618 RHLS participants were recruited to the current study. In all, seventy participants were excluded: sixty had no valid BMI recorded; nine reported a diagnosis of type 1 diabetes; and one reported using insulin. Following application of exclusion criteria, 548 participants were included in the analyses. A total of $56.1 \%$ ( $n$ 308) of participants were female, and $60.9 \%$ ( $n$ 334) of participants were classified as being overweight/obese $\left(\mathrm{BMI} \geq 27 \mathrm{~kg} / \mathrm{m}^{2}\right)$. Men had a higher fasting plasma glucose level $(5 \cdot 7$ (sD 1.1) mmol/1 v. $5 \cdot 4$ (sD 1.0) $\mathrm{mmol} / \mathrm{l}$; $P<0.001)$ and larger waist circumference $(104 \cdot 1$ (SD 12.1) $\mathrm{cm} v$. 94.1 (sD 12.6) $\mathrm{cm} ; P<0.001$ ) than women; therefore, participants were stratified by sex for all analyses. In all, eighty-two participants (15.0\%) had T2D (plasma glucose level $>7.0 \mathrm{mmol} / \mathrm{l}$ and/ or the participant self-reported taking diabetic medications). Participants with diabetes had significantly higher BMI and waist circumferences than participants without diabetes, as well as significantly higher fasting BGL, HbA1c, fasting plasma insulin levels and HOMA-IR scores (Table 1). There were no significant associations found between reported levels of physical activity and either diabetic status or $n$-3I (data not presented).

Table 1. Characteristics of participants by sex and diabetic status*

(Mean values and standard deviations)

\begin{tabular}{|c|c|c|c|c|c|c|c|c|c|c|c|c|c|c|c|}
\hline & \multicolumn{5}{|c|}{ Total (n 548) } & \multicolumn{5}{|c|}{ Men ( $n$ 240) } & \multicolumn{5}{|c|}{ Women ( $n$ 308) } \\
\hline & \multicolumn{2}{|c|}{$\begin{array}{l}\text { Diabetic }^{*} \\
(n 82)\end{array}$} & \multicolumn{2}{|c|}{$\begin{array}{l}\text { Non-diabetic } \\
(n 466)\end{array}$} & \multirow[b]{2}{*}{$P$} & \multicolumn{2}{|c|}{$\begin{array}{c}\text { Diabetic }^{*} \\
(n 44)\end{array}$} & \multicolumn{2}{|c|}{$\begin{array}{c}\text { Non-diabetic } \\
(n \text { 196) }\end{array}$} & \multirow[b]{2}{*}{$P$} & \multicolumn{2}{|c|}{$\begin{array}{c}\text { Diabetic }^{*} \\
(n 38)\end{array}$} & \multicolumn{2}{|c|}{$\begin{array}{l}\text { Non-diabetic } \\
(n 270) \\
\end{array}$} & \multirow[b]{2}{*}{$P$} \\
\hline & Mean & SD & Mean & SD & & Mean & SD & Mean & SD & & Mean & SD & Mean & SD & \\
\hline Age (years) & $76 \cdot 7$ & 5.9 & $77 \cdot 8$ & 7.1 & - & $75 \cdot 6$ & $6 \cdot 4$ & 77.5 & $6 \cdot 7$ & - & 78.0 & $5 \cdot 1$ & $78 \cdot 1$ & 7.4 & - \\
\hline Sex $(M / F)$ & \multicolumn{2}{|c|}{$44 / 38$} & \multicolumn{2}{|c|}{$196 / 270$} & - & \multicolumn{2}{|c|}{$44 / 0$} & \multicolumn{2}{|c|}{$196 / 0$} & - & \multicolumn{2}{|c|}{$0 / 38$} & \multicolumn{2}{|c|}{$0 / 270$} & - \\
\hline \multicolumn{16}{|l|}{ Ethnicity } \\
\hline Caucasian $(n)$ & \multicolumn{2}{|c|}{79} & \multicolumn{2}{|c|}{452} & - & \multicolumn{2}{|c|}{4} & \multicolumn{2}{|c|}{192} & - & \multicolumn{2}{|c|}{36} & \multicolumn{2}{|c|}{260} & - \\
\hline Aboriginal or Pacific Islander $(n)$ & \multicolumn{2}{|c|}{1} & \multicolumn{2}{|c|}{4} & - & \multicolumn{2}{|c|}{0} & \multicolumn{2}{|c|}{0} & - & \multicolumn{2}{|c|}{1} & \multicolumn{2}{|c|}{4} & - \\
\hline Asian $(n)$ & \multirow{2}{*}{\multicolumn{2}{|c|}{1}} & & & - & 0 & & 0 & & - & 1 & & 1 & & - \\
\hline Don't know/no response $(n)$ & & & $s$ & & - & 1 & & 4 & & - & 0 & & 5 & & - \\
\hline Height (m) & 1.6 & 0.1 & 1.6 & 0.1 & $<0.05$ & 1.7 & 0.1 & 1.7 & 0.1 & - & 1.6 & 0.1 & 1.6 & 0.1 & - \\
\hline Weight (kg) & 84.5 & $16 \cdot 6$ & $74 \cdot 5$ & $14 \cdot 8$ & $<0.001$ & $90 \cdot 7$ & $16 \cdot 8$ & 81.4 & $14 \cdot 2$ & $<0.001$ & $77 \cdot 2$ & $13 \cdot 3$ & 69.5 & $13 \cdot 1$ & $<0.01$ \\
\hline BMI $\left(\mathrm{kg} / \mathrm{m}^{2}\right)$ & $30 \cdot 9$ & 4.7 & $28 \cdot 1$ & 4.8 & $<0.001$ & 30.6 & 4.5 & $28 \cdot 1$ & 4.4 & $<0.01$ & 31.3 & $13 \cdot 3$ & $28 \cdot 2$ & $5 \cdot 1$ & $<0.001$ \\
\hline WC (cm) & $107 \cdot 2$ & $12 \cdot 7$ & $96 \cdot 9$ & $12 \cdot 8$ & $<0.001$ & $110 \cdot 6$ & $12 \cdot 0$ & $102 \cdot 7$ & 11.6 & $<0.001$ & $103 \cdot 7$ & $12 \cdot 7$ & $92 \cdot 7$ & 11.9 & $<0.001$ \\
\hline Fasting plasma glucose $(\mathrm{mmol} / \mathrm{l})$ & $7 \cdot 3$ & 1.4 & $5 \cdot 3$ & 0.6 & $<0.001$ & $7 \cdot 4$ & $1 \cdot 3$ & $5 \cdot 4$ & 0.6 & $<0.001$ & $7 \cdot 2$ & 1.4 & $5 \cdot 2$ & 0.5 & $<0.001$ \\
\hline HbA1c (\%) & $6 \cdot 8$ & 0.9 & $5 \cdot 7$ & 0.4 & $<0.001$ & $6 \cdot 8$ & 0.9 & $5 \cdot 7$ & 0.4 & $<0.001$ & $6 \cdot 9$ & 1.0 & $5 \cdot 7$ & 0.3 & $<0.001$ \\
\hline $\mathrm{HbA} 1 \mathrm{c}(\mathrm{mmol} / \mathrm{mol})$ & $51 \cdot 0$ & 4.0 & 39.0 & $10 \cdot 0$ & $<0.001$ & $51 \cdot 0$ & 4.0 & 39.0 & $10 \cdot 0$ & $<0.001$ & $52 \cdot 0$ & 4.0 & 39.0 & 11.0 & $<0.001$ \\
\hline Fasting insulin (mU/l) & 11.3 & $7 \cdot 4$ & $7 \cdot 3$ & 4.7 & $<0.001$ & $10 \cdot 6$ & $7 \cdot 8$ & $7 \cdot 1$ & 4.5 & $<0.001$ & $12 \cdot 1$ & 6.8 & 7.4 & 4.9 & $<0.001$ \\
\hline HOMA-IR & $3 \cdot 7$ & $2 \cdot 4$ & $1 \cdot 8$ & $1 \cdot 3$ & $<0.001$ & 3.5 & $2 \cdot 7$ & 1.8 & $1 \cdot 2$ & $<0.001$ & 3.8 & $2 \cdot 0$ & $1 \cdot 8$ & $1 \cdot 3$ & $<0.001$ \\
\hline
\end{tabular}

M/F, male/female; WC, waist circumference; HOMA-IR, homeostatic model assessment of insulin resistance.

* Diabetic status determined according to fasting blood glucose level $>7.0 \mathrm{mmol} / \mathrm{l}$ or on diabetic medications. 


\section{Macronutrient intake}

Participants' macronutrient intake is summarised in online Supplementary Table S1. There were minimal differences between the reported dietary intake of diabetic and nondiabetic participants. Protein contributed a greater proportion of energy in diabetic men than in non-diabetic men (19.6 (sD 3.3) \% v. 18.5 (sD 3.1) \%; $P<0.05$ ), and diabetic women had a significantly lower intake of carbohydrate (180.0 (SD 57.3) g $v$. 212.7 (sD 96.4) g; $P<0.05$ ) and sugars (94.8 (sD 41.8) g $v .115 \cdot 0$ (sD 57.9) g; $P<0.05$ ) compared with those without diabetes. There were no significant differences in the PUFA intake between diabetics and non-diabetics in either sex. Dietary fat, protein and carbohydrate intake had no significant correlations with fasting glucose, HbA1c, insulin or HOMA-IR (data not presented).

\section{Erythrocyte membrane fatty acid composition}

Fish oil supplement use was reported by $28 \cdot 1 \%$ ( $n$ 154) of participants. There was no significant difference between the fatty acid composition of erythrocyte membranes or markers of glycaemic control (i.e. fasting BGL, HbA1c, fasting plasma insulin and HOMA score) between those who reported consuming fish oil supplements and those who did not (data not shown), so participants were pooled for analysis. Women had a significantly higher $n$-3I than men $(8.8$ (SD 2.5$) \% \quad v .8 \cdot 1$ (sD 2.1$) \% ; P<0 \cdot 001$; data not shown). In women, diabetics had a significantly lower $n$-3I than non-diabetics (8.0 (SD $2 \cdot 0) \% v$. (Mean values and standard deviations)
8.9 (sD 2.5$) \% ; P<0.05)$, with both EPA $(20: 5 n-3)$ and DHA (22:6n-3) significantly lower in diabetics than in non-diabetics (Table 2). Oleic acid (OA; $18: 1 n$-9) was significantly higher and linoleic acid (LA; $18: 2 n-6)$ was significantly lower in diabetics when compared with non-diabetics for both sexes (Table 2).

\section{n-3 Index and markers of glycaemic control}

There was a weak but significant inverse correlation between $n$-3I and fasting plasma glucose $(r-0.092 ; P<0.05)$, as well as HbA1c $(r-0.092 ; P<0.05)$ (data not shown); however, these did not remain significant once adjusted for age and BMI (Table 3). There was a significant and proportionate decrease in the prevalence of IR as $n$-3I increased in women only (online Supplementary Table S2). When further stratified by sex and weight status, the relationship was evident only in the overweight/obese female sub-group, with no relationship between $n$-3I and IR in overweight/obese men or in the healthy/ underweight sub-group for either sex (data not presented).

\section{n-3 Index and diabetes}

Fig. 2 shows the prevalence of T2D in each quartile of $n$-3I. Among the whole group, participants with a low $n$-3I were significantly more likely to be diabetic than those with a higher $n$-3I, with OR (95\% CI) decreasing across ascending quartiles (using the lowest quartile as a reference point): 1.0 (reference), 0.95 (95\% CI $0.51,1.77), 0.72$ (95\% CI $0.38,1.39), 0.51$

Table 2. Erythrocyte membrane fatty acid (\% w/w) composition of Australian adults aged $65-95$ years by sex and diabetic status ${ }^{*}$

\begin{tabular}{|c|c|c|c|c|c|c|c|c|c|c|c|c|c|c|c|}
\hline & \multicolumn{5}{|c|}{ Total $(n 548)$} & \multicolumn{5}{|c|}{ Male $(n$ 240) } & \multicolumn{5}{|c|}{ Female $(n 308)$} \\
\hline & \multicolumn{2}{|c|}{$\begin{array}{c}\text { Diabetic } † \\
(n 82)\end{array}$} & \multicolumn{2}{|c|}{$\begin{array}{c}\text { Non-diabetic } \\
(n 466)\end{array}$} & \multirow[b]{2}{*}{$P$} & \multicolumn{2}{|c|}{$\begin{array}{c}\text { Diabetic } † \\
(n 44)\end{array}$} & \multicolumn{2}{|c|}{$\begin{array}{l}\text { Non-diabetic } \\
(n \text { 196) }\end{array}$} & \multirow[b]{2}{*}{$P$} & \multicolumn{2}{|c|}{$\begin{array}{c}\text { Diabetic } \dagger \\
(n 38)\end{array}$} & \multicolumn{2}{|c|}{$\begin{array}{l}\text { Non-diabetic } \\
(n \text { 270) }\end{array}$} & \multirow[b]{2}{*}{$P$} \\
\hline & Mean & SD & Mean & SD & & Mean & SD & Mean & $\mathrm{SD}$ & & Mean & $\mathrm{SD}$ & Mean & SD & \\
\hline$\Sigma$ SFA & $42 \cdot 2$ & 1.5 & $42 \cdot 0$ & $2 \cdot 1$ & - & $42 \cdot 4$ & 1.0 & $42 \cdot 0$ & $2 \cdot 3$ & - & 41.9 & 1.9 & $42 \cdot 1$ & $2 \cdot 0$ & - \\
\hline C16:0 & 23.0 & $1 \cdot 1$ & $22 \cdot 8$ & 1.2 & - & $23 \cdot 0$ & 0.9 & 22.9 & $1 \cdot 3$ & - & $23 \cdot 0$ & $1 \cdot 2$ & $22 \cdot 8$ & 1.2 & - \\
\hline C18:0 & 18.5 & 1.1 & $18 \cdot 6$ & 1.4 & - & $18 \cdot 7$ & $1 \cdot 1$ & 18.5 & 1.4 & - & $18 \cdot 4$ & $1 \cdot 2$ & $18 \cdot 6$ & 1.3 & - \\
\hline C20:0 & 0.7 & 0.7 & 0.6 & 0.1 & - & 0.7 & 0.7 & 0.6 & 0.2 & - & 0.6 & 0.1 & 0.6 & $0 \cdot 1$ & - \\
\hline$\Sigma$ MUFA & $18 \cdot 0$ & 1.5 & $17 \cdot 1$ & 1.6 & $<0.001$ & $17 \cdot 9$ & 1.4 & $17 \cdot 1$ & 1.6 & $<0.01$ & $18 \cdot 0$ & 1.6 & $17 \cdot 1$ & 1.6 & $<0.01$ \\
\hline C16:1n-7 & 0.6 & 0.3 & 0.6 & 0.3 & - & 0.6 & 0.3 & 0.5 & 0.3 & - & 0.7 & 0.3 & 0.6 & 0.3 & - \\
\hline C18: $1 n-9$ & $15 \cdot 2$ & $1 \cdot 2$ & $14 \cdot 4$ & 1.3 & $<0.01$ & $15 \cdot 2$ & $1 \cdot 2$ & 14.5 & $1 \cdot 3$ & $<0.01$ & $15 \cdot 2$ & $1 \cdot 3$ & $14 \cdot 4$ & 1.3 & $<0.01$ \\
\hline C18:1n-7 & 1.8 & 0.4 & $1 \cdot 7$ & 0.4 & - & 1.7 & 0.3 & 1.7 & 0.4 & - & 1.8 & 0.4 & 1.7 & 0.4 & - \\
\hline C20 : $1 n-9$ & 0.4 & 0.4 & 0.3 & 0.1 & $<0.05$ & 0.4 & 0.6 & 0.3 & 0.1 & - & 0.3 & 0.6 & 0.3 & 0.1 & - \\
\hline$\Sigma n-6$ PUFA & 27.6 & $2 \cdot 1$ & $27 \cdot 4$ & $2 \cdot 7$ & - & 27.8 & 1.9 & $27 \cdot 7$ & $2 \cdot 7$ & - & $27 \cdot 3$ & $2 \cdot 3$ & $27 \cdot 2$ & $2 \cdot 8$ & - \\
\hline C18:2n-6 & $8 \cdot 2$ & 1.4 & $8 \cdot 8$ & 1.5 & $<0.001$ & 8.4 & $1 \cdot 3$ & 9.0 & 1.5 & $<0.05$ & 8.0 & 1.4 & $8 \cdot 8$ & 1.4 & $<0.01$ \\
\hline C18:3n-6 & 0.2 & 0.1 & 0.2 & 0.2 & - & 0.2 & 0.1 & 0.2 & 0.2 & - & 0.2 & 0.2 & 0.2 & 0.2 & - \\
\hline $\mathrm{C} 20: 2 n-6$ & 0.3 & 0.3 & 0.3 & $0 \cdot 2$ & - & 0.3 & 0.4 & 0.3 & 0.3 & - & 0.3 & 0.1 & 0.3 & $0 \cdot 1$ & - \\
\hline $\mathrm{C} 20: 3 n-6$ & 1.4 & $1 \cdot 1$ & 1.5 & $1 \cdot 1$ & - & 1.7 & $1 \cdot 1$ & 1.5 & $1 \cdot 2$ & - & $1 \cdot 2$ & $1 \cdot 1$ & 1.4 & $1 \cdot 1$ & - \\
\hline C20 : 4n-6 & $17 \cdot 4$ & 2.4 & $16 \cdot 6$ & 2.5 & $<0.01$ & $17 \cdot 2$ & $2 \cdot 4$ & $16 \cdot 7$ & 1.5 & - & $17 \cdot 7$ & $2 \cdot 3$ & $16 \cdot 6$ & 2.5 & $<0.05$ \\
\hline$\Sigma n-3$ PUFA & 11.9 & $2 \cdot 3$ & $12 \cdot 4$ & 2.8 & - & 11.9 & $2 \cdot 2$ & $12 \cdot 0$ & $2 \cdot 6$ & - & 11.9 & $2 \cdot 5$ & $12 \cdot 8$ & 2.9 & - \\
\hline C18:3n-3 & 0.2 & 0.1 & 0.3 & 0.1 & - & 0.2 & 0.1 & 0.3 & 0.1 & $<0.05$ & 0.3 & 0.1 & 0.3 & 0.1 & - \\
\hline $\mathrm{C} 20: 5 n-3$ & 1.5 & 0.7 & $1 \cdot 8$ & $1 \cdot 2$ & - & $1 \cdot 4$ & 0.7 & 1.6 & 0.9 & - & 1.5 & 0.8 & 1.9 & 1.3 & $<0.05$ \\
\hline C22: $5 n-3$ & 3.6 & 0.6 & 3.6 & 0.7 & $<0.05$ & 3.6 & 0.5 & 3.6 & 0.7 & - & 3.6 & 0.7 & 3.6 & 0.7 & - \\
\hline $\mathrm{C} 22: 6 n-3$ & 6.6 & 1.4 & 6.8 & 1.5 & - & $6 \cdot 7$ & 1.4 & 6.5 & 1.4 & - & 6.5 & 1.4 & $7 \cdot 0$ & 1.4 & $<0.05$ \\
\hline$n-3 \mid \ddagger$ & 8.1 & 1.9 & 8.6 & 2.4 & - & $8 \cdot 1$ & 1.9 & 8.0 & $2 \cdot 1$ & - & 8.0 & $2 \cdot 0$ & 8.9 & 2.5 & $<0.05$ \\
\hline
\end{tabular}

HOMA-IR, homeostatic model assessment of insulin resistance; -, NS; $n$-3I, $n$-3 index.

* Group differences between diabetics and non-diabetics were assessed using independent-samples $t$ tests (two-tailed)

† Diabetic status determined according to fasting blood glucose level $>7.0 \mathrm{mmol}$ or on diabetic medications ${ }^{(19)}$.

$\ddagger n-3 \mathrm{I}=$ erythrocyte EPA $\%+$ DPA\% of total fatty acids ${ }^{(18)}$. 
Table 3. Correlations between erythrocyte fatty acids (\% w/w) and markers of glycaemic control in Australian adults aged $65-95$ years, adjusted for age and $\mathrm{BMI}^{*}$

\begin{tabular}{|c|c|c|c|c|c|c|c|c|}
\hline & \multicolumn{2}{|c|}{ Fasting plasma glucose (mmol/l) } & \multicolumn{2}{|c|}{ Fasting plasma insulin (mU/l) } & \multicolumn{2}{|c|}{ HbA1c (\%) } & \multicolumn{2}{|c|}{ HOMA-IR† } \\
\hline & $r$ & $P$ & $r$ & $P$ & $r$ & $P$ & $r$ & $P$ \\
\hline$\Sigma$ SFA & -0.007 & - & 0.024 & - & -0.042 & - & 0.027 & - \\
\hline C16:0 & -0.033 & - & -0.037 & - & -0.031 & - & -0.018 & - \\
\hline C18:0 & -0.042 & - & 0.075 & - & -0.054 & - & 0.062 & - \\
\hline C20: 0 & 0.013 & - & -0.021 & - & 0.077 & - & -0.021 & - \\
\hline$\Sigma$ MUFA & 0.140 & $<0.01$ & 0.061 & - & 0.117 & $<0.01$ & 0.094 & $<0.05$ \\
\hline C16:1n-7 & 0.006 & - & 0.031 & - & -0.088 & $<0.05$ & 0.023 & - \\
\hline C18:1n-9 & 0.181 & $<0.001$ & 0.082 & - & 0.174 & $<0.001$ & 0.125 & $<0.01$ \\
\hline C18:1n-7 & -0.062 & - & -0.034 & - & -0.074 & - & -0.041 & - \\
\hline C20: $1 n-9$ & 0.044 & - & -0.032 & - & 0.053 & - & -0.024 & - \\
\hline$\Sigma n-6$ PUFA & -0.001 & - & 0.000 & - & 0.039 & - & 0.006 & - \\
\hline C18:2n-6 & -0.147 & $<0.01$ & -0.093 & $<0.05$ & -0.093 & $<0.05$ & -0.119 & $<0.01$ \\
\hline C18:3n-6 & 0.020 & - & -0.033 & - & -0.034 & - & 0.011 & - \\
\hline $\mathrm{C} 20: 2 n-6$ & 0.004 & - & -0.075 & - & 0.004 & - & -0.052 & - \\
\hline $\mathrm{C} 20: 3 n-6$ & 0.009 & - & -0.011 & - & 0.058 & - & -0.013 & - \\
\hline $\mathrm{C} 20: 4 n-6$ & 0.080 & - & 0.069 & - & 0.071 & - & 0.084 & 0.05 \\
\hline$\Sigma n-3$ PUFA & -0.063 & - & 0.000 & - & -0.092 & $<0.05$ & -0.014 & - \\
\hline C18:3n-3 & -0.063 & - & -0.020 & - & -0.075 & - & -0.029 & - \\
\hline $\mathrm{C} 20: 5 n-3$ & -0.075 & - & -0.024 & - & -0.092 & $<0.05$ & -0.039 & - \\
\hline $\mathrm{C} 22: 5 n-3$ & 0.020 & - & -0.056 & - & -0.072 & - & -0.032 & - \\
\hline $\mathrm{C} 22: 6 n-3$ & -0.066 & - & 0.048 & - & -0.063 & - & 0.022 & - \\
\hline$n-3 \mid \ddagger$ & -0.077 & - & 0.018 & - & -0.083 & - & -0.005 & - \\
\hline
\end{tabular}

HOMA-IR, homeostatic model assessment of insulin resistance; -, NS; $n$-3I, $n$-3 index.

* All correlations were assessed using Pearson's coefficient and significance determined using two-tailed $t$ tests, adjusted for age and BMI.

† HOMA-IR = fasting blood glucose $\times$ fasting plasma insulin/22.5.

$\ddagger n-3 \mathrm{l}=$ erythrocyte EPA $\%+$ DPA $\%$ of total fatty acids $^{(18)}$.

(95\% CI $0.25,1.03)\left(P_{\text {trend }}<0.05\right)$. Adjusting for age and BMI did not markedly change these values. When stratified by sex, there was a significant inverse relationship between $n$-3I and T2D in women, with OR again decreasing across ascending quartiles: $1 \cdot 0$ (reference), $0 \cdot 81$ (95\% CI 0.33, 2.01), 0.56 (95\% CI $0.23,1.37), 0.26$ (95\% CI 0.09, 0.82) ( $\left.P_{\text {trend }}<0.01\right)$. However, no significant relationship was detected between $n-3 \mathrm{I}$ and T2D in men, with an OR across ascending quartiles of 1.0 (reference), 0.93 (95\% CI $0.36,2.39), 0.95$ (95\%CI 0.37, 2.43) and $0.93(0.33$, $2.58)\left(P_{\text {trend }}=0.903\right)$. When further stratified by weight status, there was no significant relationship evident between $n$-3I and diabetes in the healthy/underweight sub-group for either sex, although in healthy/underweight men there was a nonsignificant positive association between T2D and $n$-3I with an OR across quartiles of 1.0 (reference), 1.24 (95\% CI $0.2,9 \cdot 4$ ), 1.72 (95\% CI $0.2,13.3), 5.2$ (95\% CI 0.8, 32.0) $\left(P_{\text {trend }}=0.067\right)$. There was a significant inverse association in overweight/obese women $\left(\mathrm{BMI} \geq 27 \mathrm{~kg} / \mathrm{m}^{2}\right.$ ), with an OR of 1.0 (reference), 0.82 (95\% CI $0.31,2 \cdot 18), 0.56(95 \%$ CI $0.21,1.52)$ and 0.22 (95\% CI $0.06,0.82)$ in the first to fourth quartiles, respectively $\left(P_{\text {trend }}<0.05\right)$. Overweight/obese men showed a similar trend, with an OR across quartiles of 1.0 (reference), 0.86 (95\% CI $0.32,2 \cdot 29), 0.76$ (95\% CI $0.25,2.33)$ and 0.46 (95\% CI 0.14 , 1.54); however, this finding did not reach significance $\left(P_{\text {trend }}=0 \cdot 211\right)$.

When considered independently, neither EPA nor DHA was significantly associated with T2D in women in either the healthy weight or overweight/obese subgroups. Among men, a higher erythrocyte EPA was associated with a decrease in the odds of having T2D, with an OR of 0.533 (95\%CI 0.283, 0.936;
$P<0.05)$ for every 1 unit increase in EPA. Conversely, a higher DHA was associated with an increased odds of T2D, with an OR of 1.427 (95\%CI $0.1012,2.013 ; P=0.042$ ) for every 1 unit increase in erythrocyte DHA. When split into healthy and overweight/obese subgroups, the only significant association that remained was between DHA and T2D in healthy-weight men, with an OR of 2.789 (95\%CI $1.255,6 \cdot 197 ; P=0.012)$ for every 1 unit increase in DHA.

\section{Discussion}

This study set out to determine whether erythrocyte $n$-3 PUFA status, as measured by the $n-3 \mathrm{I}$, is associated with T2D in a population of older adults, and to test the hypothesis that the $n$-3I is inversely correlated with IR in an overweight subgroup. Our results show a significant inverse relationship between $n$ - $3 \mathrm{I}$ and T2D in overweight/obese women, but not in men. To date, the literature articulating a role of dietary marine-based $n-3$ PUFA in the development of T2D has been unclear. Multiple systematic reviews have provided inconsistent findings, with one concluding that dietary $n$-3 PUFA intake increases the risk of $\mathrm{T} 2 \mathrm{D}^{(4)}$, whereas two have reported an inverse association $^{(5,7)}$, and a further two showed no association ${ }^{(6,8)}$. The majority of studies included in these reviews used questionnaires to determine dietary intake of $n-3$ PUFA, which may be prone to both reporting and measurement errors. One study that did compare objective biomarkers of dietary n-3 PUFA intake with the prevalence of T2D and markers of glycaemic control in older adults reported that a higher concentration of 
(a)

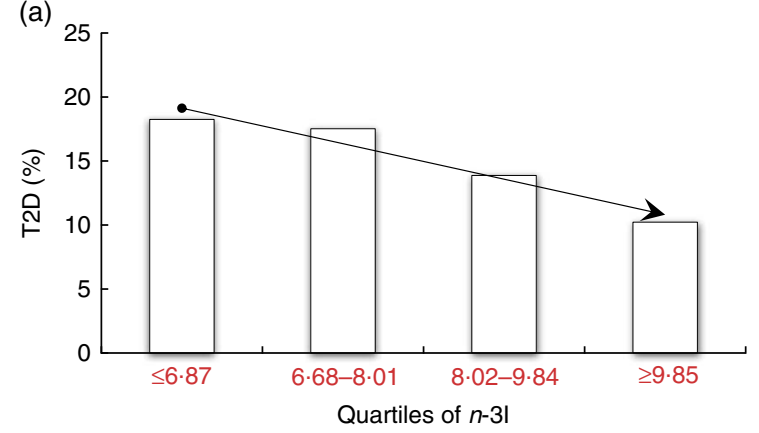

(b)

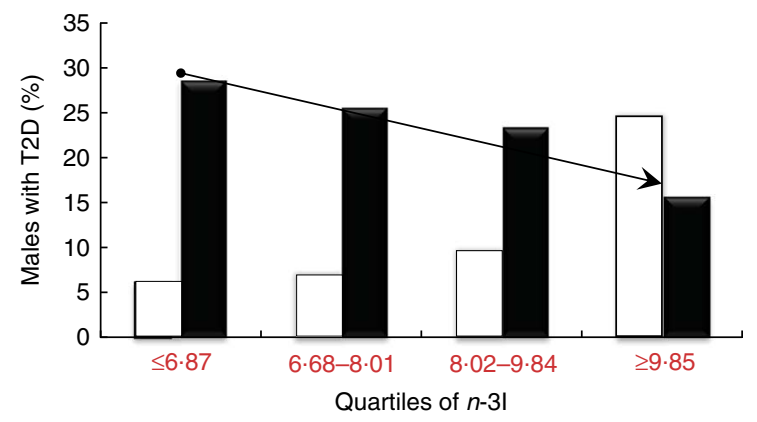

(c)

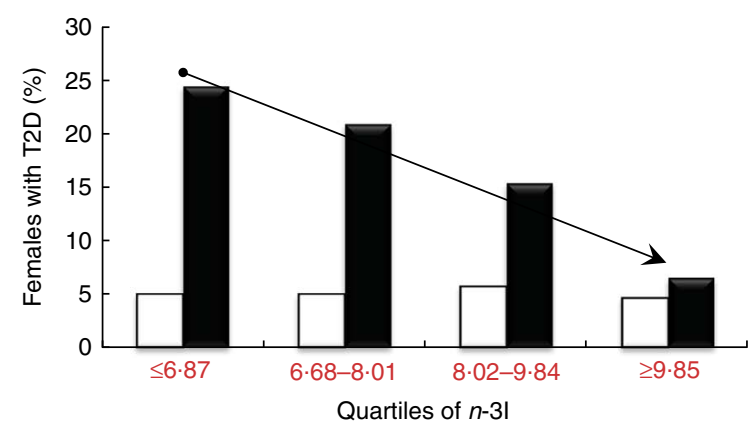

Fig. 2. (a) Prevalence of type 2 diabetes (T2D) in each quartile of $n-3$ index $(n-3 \mathrm{l})$. (b) Percentage of males with T2D in each quartile of $n-3 \mathrm{l}$ according to weight status. (c) Percentage of females with T2D in each quartile of $n-3$ according to weight status. Diabetic status determined according to fasting blood glucose level $>7.0 \mathrm{mmol}$ or on diabetic medications ${ }^{\left({ }^{19)}\right)} . n-3 \mathrm{l}=$ erythrocyte EPA\% + DPA\% of total fatty acids ${ }^{(18)}$. Trends in proportions assessed using linear-by-linear association. a: $\square$, Total population; b,c: $\square$, healthy/ underweight $\left(\mathrm{BMI}<27 \mathrm{~kg} / \mathrm{m}^{2}\right) ; \boldsymbol{\square}$, overweight/obese $\left(\mathrm{BMI} \geq 27 \mathrm{~kg} / \mathrm{m}^{2}\right)$.

plasma EPA and DHA was associated with a lower risk of incident diabetes ${ }^{(21)}$. Furthermore, a recent study in pre-diabetic men and women found baseline serum EPA and DHA to be associated with a reduced incidence of T2D over an 11-year follow-up ${ }^{(22)}$. Conversely, Mahendran et al. ${ }^{(23)}$ did not find a relationship between erythrocyte $n-3$ PUFA and incident diabetes in a large prospective cohort study of middle-aged men over a 5-year follow-up. Similar to our findings, Zhang et al. ${ }^{(24)}$ found that plasma levels of DHA were inversely correlated with the presence of IR in women $(P=0.03)$ but not in men. Results of the present study highlight differences in response based on both weight status and sex, a novel finding that provides direction for future research.
In the current study, no direct correlation between $n$-3I and HOMA-IR score in the overweight sub-group was found; however, those in the highest quartile of $n$-3I were the least likely to be categorised as insulin resistant. The $n$-3I has been shown to be negatively correlated with HOMA-IR scores in children with obesity ${ }^{(11)}$, whereas no correlation was found in predominately healthy-weight adolescents ${ }^{(25)}$, consistent with a weight-dependent effect. Supporting this, randomised control trials in obese children, adolescents and women have found $n-3$ PUFA supplementation to reduce $\operatorname{IR}^{(26,27)}$. Conversely, some studies using high-dose $n$-3 PUFA supplementation $(>2 \cdot 5-3 \mathrm{~g} / \mathrm{d})$ have found no affect on insulin sensitivity ${ }^{(28,29)}$. These findings collectively suggest that potential benefits of $n-3$ PUFA in glycaemic control may be limited to overweight populations, and regular low dose EPA and DHA provided through habitual dietary intake, rather than short-term high dose supplementation, may be of optimal benefit.

It should be noted that the mean $n$-3I values obtained in this study are considerably higher than previous studies have reported $^{(10)}$. This could possibly be because of the older age of our participants, as the $n$-3I has been shown to increase with age ${ }^{(30)}$. As the dietary data collected for this study did not include a specific breakdown of $n$-3 PUFA intake, it cannot be ruled out that a higher mean $n$-3I was because of a high intake of fish or seafood, or a higher intake of $n$-3 PUFA supplements. It is also possible that the high $n$ - 3 I values are because of minor differences in the technique used to obtain and calculate the $n$-3I by different laboratories. Although the $n$-3I values from this study might not be directly comparable to previous studies, the relationships and trends found are valid and findings are robust.

A number of significant differences in the erythrocyte membrane fatty acid composition between diabetics and nondiabetics were noted. LA (C18:2n-6) was significantly lower in diabetic individuals, whereas arachidonic acid (C20:4n-6) and oleic acid (OA; C18: $1 n$-9) were significantly higher, possibly reflecting an up-regulation of stearoyl-CoA desaturase and $\Delta-6$ desaturase enzyme activities ${ }^{(31)}$. The $n$-3I was significantly lower in diabetic compared with non-diabetic women, with both EPA and DHA content showing significant differences. Women in our study had a significantly higher $n$-3I than men, consistent with literature that women have a greater ability to synthesise EPA and DHA from their precursor $\alpha$-linolenic acid than men ${ }^{(32)}$. Sex differences in the physiological response to EPA and DHA supplementation have been reported in previous metabolic studies ${ }^{(33,34)}$, although not directly in studies that included measures of glycaemia or diabetes.

Although a relationship between the $n$-3I and T2D was evident in women only, when results were further split into either a healthy weight $\left(\mathrm{BMI}<27 \mathrm{~kg} / \mathrm{m}^{2}\right)$ or overweight/obese (BMI $\geq 27 \mathrm{~kg} / \mathrm{m}^{2}$ ) subgroup there was no significant relationship in the healthy-weight group for either sex. Although the inverse association between $n$-3I and T2D was only significant in women who were overweight or obese, men displayed a similar trend, with the prevalence of T2D in the highest quartile for $n$-3I being half that of the lowest quartile. Interestingly, although non-significant, $n$-3I was positively associated with T2D in healthy/underweight men. Potential mechanisms for the differing relationships for the $n-3 \mathrm{I}$ and T2D between healthy 
and overweight/obese men are currently unknown. Findings from this study suggest that DHA is likely to play a significant role, with a more than 2-fold increase seen in the odds of having T2D for every 1 unit increase of erythrocyte DHA seen in healthy-weight, but not overweight/obese, men. The lack of significance found for the association between $n-3 \mathrm{I}$ and T2D in healthy-weight men may be because of the small number of healthy-weight men in our study, and further studies with a larger sample of healthy-weight men should be conducted to confirm this finding, as well as mechanistic studies to elucidate potential mechanisms for why DHA is positively associated with T2D in healthy, but not overweight/obese, men. Overall, these results suggest that a higher $n-3$ PUFA status may be a contributing factor to metabolically healthy obesity.

There were few significant differences in macronutrient intake between diabetics and non-diabetics for either sex. Given that individuals with a high $n$-3I have previously been shown to be generally health conscious and consume diets high in fruits, vegetables and wholegrain cereals ${ }^{(35)}$, which is known to be protective for the development of $\mathrm{T} 2 \mathrm{D}^{(36)}$, it is crucial to determine whether there is a causal relationship between $n$-3I and T2D. Given that there were few differences between the macronutrient intake of the diabetic and non-diabetic groups, it is likely that there is an independent mechanism implicated.

Although adiponectin was not assessed as part of this study, this adipokine is involved in the insulin signalling pathway and may play a role in the development of obesity-related $\mathrm{IR}^{(37)}$. As body fat increases, adiponectin levels decrease, which correlates with increasing levels of IR. Moreover, increasing adiponectin levels have been demonstrated to increase insulin sensitivity ${ }^{(37)}$. Fish oils rich in $n$-3 PUFA have demonstrated an ability to increase adiponectin levels ${ }^{(38)}$, with EPA and DHA acting as ligands for PPAR $\gamma$, stimulating adiponectin transcription $^{(39)}$. As adiponectin decreases with increasing levels of obesity, this may be why the $n$-3I is negatively associated with T2D in overweight/obese, but not healthy-weight, men and women. The modulation of adiponectin by $n-3$ PUFA via PPAR $\gamma$ offers a biologically plausible mechanism by which a higher $n-3$ PUFA status results in a reduced prevalence of T2D in overweight individuals, and adiponectin should be included in future studies.

This analysis has some limitations. HOMA-IR is considered to be the least sensitive method of evaluating IR, and results may be different if more sensitive methods, such as the hyperinsulinaemic euglycaemic clamp had been utilised in the study. Other limitations include a lack of specific $n-3$ PUFA dietary data collected. However, this was overcome through the use of objective biomarkers; cross-sectional study design, which means that a causal relationship between $n-3$ PUFA status and T2D cannot be drawn; and primarily Caucasian study population, which limits transferability. However, this study also had some strengths, including a large sample size and biochemical data collection in a population of independently living older adults. The proportions of women and diabetics included in the study are comparable to those reported for people over 65 years of age within the general Australian community, making this a somewhat representative sample.

\section{Conclusion}

There is a significant inverse relationship between $n$-3 PUFA status and T2D in overweight/obese women, with a similar trend noted in overweight/obese men. However, higher $n$-3 PUFA status has a positive relationship with T2D in healthy-weight men. Although a causal relationship cannot be drawn from this cross-sectional study, results support a potential role for dietary $n-3$ PUFA intake in improving the metabolic health of individuals with obesity. Further investigations are warranted to elucidate sex-based differences and to determine whether dietary interventions to improve $n-3$ PUFA status can prevent the onset of T2D in at-risk individuals

\section{Acknowledgements}

The research on which this paper is based was conducted as part of the RHLS, The University of Newcastle. We wish to acknowledge the involvement of the members of the Retirement Health and Lifestyle Research Group. We are grateful to the men and women of the Central Coast region who participated in the study. The authors would also like to acknowledge Melissa Fry for her assistance in the fatty acid analysis.

The RHLS was funded by an ARC Linkage Project Grant (LP0883378). Funding and in-kind support was also received from the Northern Sydney Central Coast Health Public Health Unit, Uniting Care Ageing NSW. ACT, Urbis Pty Ltd and Valhalla Village Pty Ltd and in-kind support was provided by the Hunter Valley Research Foundation and Lend Lease. Funding bodies had no role in the design, analysis or writing of this manuscript.

M. L. G., M. V. and M. L. designed the research; S. N., K. K. and K. A. A. conducted the research; K. A. A. analysed the data; K. A. A., M. L. G. and T. B. wrote the paper; and K. A. A. had primary responsibility for final content. All authors read and approved the final manuscript.

The authors declare that there are no conflicts of interest.

\section{Supplementary material}

For supplementary material/s referred to in this article, please visit http://dx.doi.org/doi:10.1017/S0007114516000258

\section{References}

1. Wild S, Roglic G, Green A, et al. (2004) Global prevalence of diabetes: estimates for the year 2000 and projections for 2030 . Diabetes Care. 27, 1047-1053.

2. Malekshahi Moghadam A, Saedisomeolia A, Djalali M, et al. (2012) Efficacy of omega-3 fatty acid supplementation on serum levels of tumour necrosis factor-alpha, C-reactive protein and interleukin- 2 in type 2 diabetes mellitus patients. Singapore Med J 53, 615-619.

3. Hartweg J, Perera R, Montori V, et al. (2008) Omega-3 polyunsaturated fatty acids (PUFA) for type 2 diabetes mellitus. Cochrane Database Syst Rev Issue 1. Art. No. CD003205.

4. Zhou Y, Tian C \& Jia C (2012) Association of fish and n-3 fatty acid intake with the risk of type 2 diabetes: a meta-analysis of prospective studies. Br J Nutr 108, 408-417.

5. Wallin A, Di Giuseppe D, Orsini N, et al. (2012) Fish consumption, dietary long-chain $n$-3 fatty acids, and risk of type 2 diabetes: systematic review and meta-analysis of prospective studies. Diabetes Care 35, 918-929. 
6. Zhang M, Picard-Deland E \& Marette A (2013) Fish and marine omega-3 polyunsaturated fatty acid consumption and incidence of type 2 diabetes: a systematic review and meta-analysis. Int J Endocrinol 2013, 501015.

7. Zheng JS, Huang T, Yang J, et al. (2012) Marine $N-3$ polyunsaturated fatty acids are inversely associated with risk of type 2 diabetes in Asians: a systematic review and meta-analysis. PLOS ONE 7, e44525.

8. Wu JH, Micha R, Imamura F, et al. (2012) Omega-3 fatty acids and incident type 2 diabetes: a systematic review and meta-analysis. Br J Nutr 107, Suppl. 2, S214-S227.

9. Nkondjock A \& Receveur O (2003) Fish-seafood consumption, obesity, and risk of type 2 diabetes: an ecological study. Diabetes Metab 29, 635-642.

10. Harris WS (2009) The omega-3 index: from biomarker to risk marker to risk factor. Curr Atheroscler Rep 11, 411-417.

11. Burrows T, Collins CE \& Garg ML (2011) Omega-3 index, obesity and insulin resistance in children. Int J Pediatr Obes $\mathbf{6}$, e532-e539.

12. Lassale C, Guilbert C, Keogh J, et al. (2009) Estimating food intakes in Australia: validation of the Commonwealth Scientific and Industrial Research Organisation (CSIRO) food frequency questionnaire against weighed dietary intakes. J Hum Nutr Diet 22, 559-566.

13. Giles-Corti B, Timperio A, Cutt H, et al. (2006) Development of a reliable measure of walking within and outside the local neighbourhood: RESIDE's Neigbourhood Physical Activity Questionnaire. Prev Med 42, 455-459.

14. Craig C, Marshall A, Sjostrom M, et al. (2003) International physical activity questionnaire: 12-country reliability and validity. Med Sci Sports Exerc 35, 1381-1395.

15. Australian Institute of Health and Welfare (2003) The Active Australia Survey: A Guide and Manual for Implementation, Analysis and Reporting. Canberra: Australian Institute of Health and Welfare.

16. Janssen I \& Mark AE (2007) Elevated body mass index and mortality risk in the elderly. Obesity Rev $\mathbf{8}, 41-59$.

17. Lepage G \& Roy CC (1986) Direct transesterification of all classes of lipids in a one-step reaction. J Lipid Res 27, 114-120.

18. Harris WS (2007) Omega-3 fatty acids and cardiovascular disease: a case for omega-3 index as a new risk factor. Pharmacol Res 55, 217-223.

19. World Health Organisation (2006) Definition and Diagnosis of Diabetes Mellitus and Intermediate Hyperglycemia. Geneva: WHO Document Production Services.

20. Esteghamati A, Ashraf H, Esteghamati A-R, et al. (2009) Optimal threshold of homeostasis model assessment for insulin resistance in an Iranian population: the implication of metabolic syndrome to detect insulin resistance. Diabetes Res Clin Pract 84, 279-287.

21. Djousse L, Biggs ML, Lemaitre RN, et al. (2011) Plasma omega-3 fatty acids and incident diabetes in older adults. $A m J$ Clin Nutr 94, 527-533.

22. Takkunen MJ, Schwab US, de Mello VD, et al. (2015) Longitudinal associations of serum fatty acid composition with type 2 diabetes risk and markers of insulin secretion and sensitivity in the Finnish Diabetes Prevention Study. Eur J Nutr (epublication ahead of print version 1 May 2015)

23. Mahendran Y, Agren J, Uusitupa M, et al. (2014) Association of erythrocyte membrane fatty acids with changes in glycemia and risk of type 2 diabetes. Am J Clin Nutr 99, 79-85.
24. Zhang G, Sun Q, Hu FB, et al. (2012) Erythrocyte n-3 fatty acids and metabolic syndrome in middle-aged and older Chinese. J Clin Endocrinol Metab 97, E973-E977.

25. Lauritzen L, Harslof LB, Hellgren LI, et al. (2012) Fish intake, erythrocyte $n-3$ fatty acid status and metabolic health in Danish adolescent girls and boys. Br J Nutr 107, 697-704.

26. Juarez-Lopez C, Klunder-Klunder M, Madrigal-Azcarate A, et al. (2013) Omega-3 polyunsaturated fatty acids reduce insulin resistance and triglycerides in obese children and adolescents. Pediatr Diabetes 14, 377-383.

27. Rafraf M, Mohammadi E, Asghari-Jafarabadi M, et al. (2012) Omega-3 fatty acids improve glucose metabolism without effects on obesity values and serum visfatin levels in women with polycystic ovary syndrome. J Am Coll Nutr 31, 361-368.

28. Crochemore IC, Souza AF, de Souza AC, et al. (2012) $\omega-3$ Polyunsaturated fatty acid supplementation does not influence body composition, insulin resistance, and lipemia in women with type 2 diabetes and obesity. Nutr Clin Pract 27, $553-560$

29. Kelley DS, Adkins Y, Woodhouse LR, et al. (2012) Docosahexaenoic acid supplementation improved lipocentric but not glucocentric markers of insulin sensitivity in hypertriglyceridemic men. Metab Syndr Relat Disord 10, 32-38.

30. Harris WS, Pottala JV, Varvel SA, et al. (2013) Erythrocyte omega-3 fatty acids increase and linoleic acid decreases with age: observations from 160,000 patients. Prostaglandins Leukot Essent Fatty Acids 88, 257-263.

31. Kroger J, Zietemann V, Enzenbach C, et al. (2011) Erythrocyte membrane phospholipid fatty acids, desaturase activity, and dietary fatty acids in relation to risk of type 2 diabetes in the European Prospective Investigation into Cancer and Nutrition (EPIC)-Potsdam Study. Am J Clin Nutr 93, 127-142.

32. Childs CE, Romeu-Nadal M, Burdge GC, et al. (2008) Gender differences in the $n-3$ fatty acid content of tissues. Proc Nutr Soc 67, 19-27.

33. Phang M, Sinclair AJ, Lincz LF, et al. (2012) Gender-specific inhibition of platelet aggregation following omega-3 fatty acid supplementation. Nutr Metab Cardiovasc Dis 22, 109-114.

34. Phang M, Lincz LF \& Garg ML (2013) Eicosapentaenoic and docosahexaenoic acid supplementations reduce platelet aggregation and hemostatic markers differentially in men and women. J Nutr 143, 457-463.

35. O'Sullivan TA, Ambrosini GL, Mori TA, et al. (2011) Omega-3 index correlates with healthier food consumption in adolescents and with reduced cardiovascular disease risk factors in adolescent boys. Lipids 46, 59-67.

36. Colagiuri R, Girgis S, Gomez M, et al. (2009) National Evidence Based Guidelines for the Primary Prevention of Type 2 Diabetes. Canberra: National Health and Medical Research Council.

37. Kadowaki T, Yamauchi T, Kubota N, et al. (2006) Adiponectin and adiponectin receptors in insulin resistance, diabetes, and the metabolic syndrome. J Clin Invest 116, 1784-1792.

38. Gray B, Steyn F, Davies PS, et al. (2013) Omega-3 fatty acids: a review of the effects on adiponectin and leptin and potential implications for obesity management. Eur J Clin Nutr 67, 1234-1242.

39. Grygiel-Gorniak B (2014) Peroxisome proliferator-activated receptors and their ligands: nutritional and clinical implications - a review. Nutr J 13, 17. 